\title{
Sagnac Effect in Coupled-Resonator Slow-Light Waveguide Structures
}

\author{
Jacob Scheuer ${ }^{1, *}$ and Amnon Yariv ${ }^{2}$ \\ ${ }^{1}$ Center for the Physics of Information, California Institute of Technology, M/C 128-95, Pasadena, California 91125, USA \\ ${ }^{2}$ Department of Applied Physics, California Institute of Technology, Pasadena, California 91125, USA
}

(Received 25 April 2005; published 6 February 2006)

\begin{abstract}
We study the effect of rotation on the propagation of electromagnetic waves in slow-light waveguide structures consisting of coupled microring resonators. We show that such configurations exhibit a new type of Sagnac effect which can be used for the realization of highly compact integrated rotation sensors and gyroscopes.
\end{abstract}

When an electromagnetic wave propagates in a moving medium it accumulates additional phase shift, compared to a wave propagating in a stationary medium, which depends on the scalar product of the wave propagation direction and the velocity vector of the medium [1,2]. A particularly interesting configuration is that of a wave propagating along a circular path in a rotating medium. In such a scenario, the additional phase accumulated by the wave depends on the relation between the propagation directions of the medium and the wave (codirectional or counterdirectional). This phase difference is often referred to as the Sagnac effect and in addition to its scientific importance, it has numerous practical applications such as detection and high-precision measurement of rotation.

In the past few years, much attention has been devoted to slowing down the propagation speed of light and to coherently stop and store pulses of light [3-6]. There are two major approaches to achieve significant reduction of the group velocity of light, which employ either electronic or optic resonances. Because of the inherent constraints associated with the conversion of the optical signals to coherent electronic states, the electronic resonance approach is less attractive for practical implementations of slow-light devices. Consequently, significant efforts were focused on controlling the speed of light using photonic structures incorporating microcavities and photonic crystals. Substantial delays and storage of light pulses were predicted in various coupled-cavities structures such as coupled-resonator optical waveguides (CROWs) [7] and side-coupled integrated spaced sequence of resonators (SCISSORs) [8]. In addition to their practical advantages, coupled-cavity waveguides (CCW) exhibit similar characteristics to those of periodic atomic lattices, such as energy (frequency) bands, forbidden gaps, etc., This property makes optical periodic structures, and, in particular, $\mathrm{CCW}$, an excellent platform for study and demonstration of the physical phenomena found and predicted in atomic systems such as band anticrossing and electromagnetically induced transparency (EIT) [7].

Recently, Leonhardt and Piwnitski pointed out the advantages of using the Sagnac effect in slow-light medium generated by EIT for the realization of an ultrasensitive optical gyroscope [9]. Subsequently, Steinberg studied the effect of rotation in coupled photonic crystal defect cavities [10] and Matsko et al. proposed to utilize the dispersive characteristics of slow-light propagation in a closedloop SCISSOR-like configuration to realize a highsensitivity miniaturized optical gyroscope [11]. In that study, however, the SCISSOR was modeled as a highlydispersive conventional waveguide where the slow group velocity of the light in the SCISSOR stems from the average interaction of the light with the high- $Q$ resonators.

In this Letter, we study the properties of the Sagnac effect in a CROW which is wrapped around itself, with application for a highly compact rotation sensor or an optical gyroscope. Figure 1 illustrates the geometrical configuration: light is launched into the input waveguide and equally divided between the two channels of the $3 d B$ coupler. The signal in each arm is coupled to a different end of the circular CROW consisting of directly coupled ring resonators. Finally, the counterpropagating signals (marked by the gray and white arrows) are combined by the $3 d B$ coupler where the output signal in each arm of the coupler depends on the relative phase difference between the signals:

$$
\begin{aligned}
& \left|A_{r}\right|^{2}=\left|E_{\text {in }}\right|^{2} \cos ^{2}\left(\frac{1}{2} \Delta \phi\right) ; \\
& \left|B_{r}\right|^{2}=\left|E_{\text {in }}\right|^{2} \sin ^{2}\left(\frac{1}{2} \Delta \phi\right),
\end{aligned}
$$

where $E_{\text {in }}$ and $\Delta \phi$ are correspondingly the input amplitude and the phase difference between the counterrotating

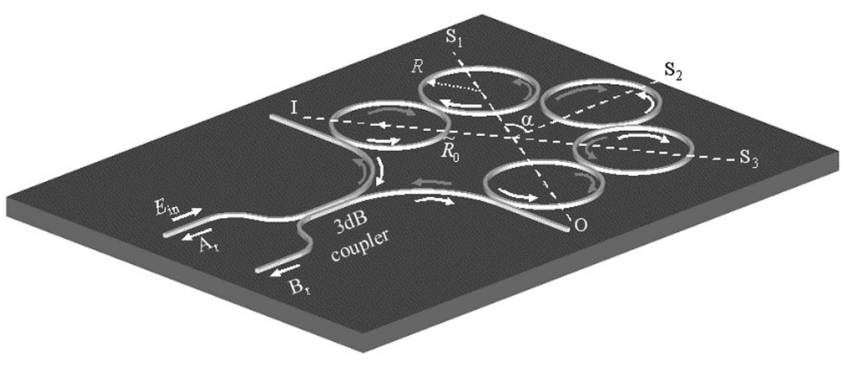

FIG. 1. Schematic of the coupled-resonator slow-light rotation sensor. 
fields. When the device is stationary, the overall phases accumulated by both signals are identical, i.e., $\Delta \phi=0$, resulting in complete cancellation of $B_{r}$. On the other hand, when the device is rotating, the phases accumulated by the signals differ, resulting in a nonvanishing intensity $B_{r}$.

To evaluate the phase difference $\Delta \phi$ in a CROW it is convenient to divide the structure into sections as illustrated in Fig. 1: An input section which consists of the CROW input coupler and part of the first microring (this section is marked by the dashed white line "I"). A recurring section consisting of two halves of a microring coupled to a complete ring, constituting the main body of the CROW (this section is defined by two successive dashed white lines: $I \rightarrow S_{1}, S_{1} \rightarrow S_{2}$, etc.). And an output section which is similar to the input section (from the line marked by "O" to the output coupler). Because of the recurring section, it is convenient to represent each section by a transfer matrix linking between the input and output ports of the section. The overall transfer matrix of the structure is then found simply by multiplying these matrices in the correct order.

The phase accumulated by a wave propagating in nonstationary waveguide depends primarily on the scalar product of the waveguide velocity and the wave vector $k$. In the configuration studied here, the contribution of each segment $d \vec{r}$ in each microring is different because the center of rotation does not necessarily coincide with the center of any of the microrings. Therefore, in order to construct the transfer matrix of each section we have to evaluate the phase accumulated by a wave propagating along a curved waveguide segment which is rotating around an arbitrary point.

Figure 2 illustrate the geometry of this problem: a wave propagating in microring resonator with radius $R$ while the center of this ring is rotating with angular velocity $\Omega$ around a fixed point. The distance between the center of the microring and the center of rotation is $\tilde{R}_{0}$. The phase accumulated by the wave as it propagates along a segment $d \vec{r}$ stems from two contributions: The conventional phase due to the propagation $d\left(\Delta \phi_{\text {prop }}\right)=\omega / c n|d r|$ and the rotation-related phase shift which is given by [1]:

$$
d\left(\Delta \phi_{\mathrm{rot}}\right)=\frac{\omega n^{2}}{c^{2}}(1-\gamma) \vec{V} \cdot d \vec{r},
$$

where $\omega$ is the optical (angular) frequency, $c$ is the speed of light in vacuum, $n$ is the refractive index, $\vec{V}=\vec{\Omega} \times\left(\overrightarrow{\tilde{R}}_{0}+\right.$ $\vec{R})$ is the linear velocity of the segment, and $\gamma$ is the Fresnel-Fizeau drag coefficient given by $\gamma=c\left(1-n^{-2}\right)$ (for a nondispersive medium). Therefore, the overall rotation-related phase accumulated by an electromagnetic wave which propagates in a microring from $\theta_{s}$ to $\theta_{f}$ (see Fig. 2) is given by:

$$
\begin{aligned}
\Delta \phi_{\mathrm{rot}} & =\int_{\theta_{s}}^{\theta_{f}} d\left(\Delta \phi_{\mathrm{rot}}\right) \\
& =\frac{\omega \Omega}{c^{2}} R^{2}\left(\theta_{f}-\theta_{s}\right)+\frac{\omega \Omega}{c^{2}} R \tilde{R}_{0}\left(\cos \theta_{f}-\cos \theta_{s}\right) .
\end{aligned}
$$

Equation (3) exhibits several interesting properties that should be noted. First, the rotation-related phase shift is independent of the waveguide index of refraction $n$. This is a well-known property of the Sagnac effect which does not depend on the refractive index of the medium comprising the loop. Second, for a complete loop the second term in (3) vanishes and thus the phase shift is independent of the center of the rotation. However, in the structure analyzed here, the propagation section between two couplers does not form a complete loop, and therefore, the second term must be included.

For simplicity, we assume that the microrings are identical and lossless and that the coupling coefficients $\kappa$ between adjacent microrings are also identical. The transfer matrices for the three types of sections are straightforwardly given by

$$
\begin{aligned}
& M_{1}=\left(\begin{array}{cc}
0 & \operatorname{expi}\left(\phi_{P}^{\alpha / 2}+D \phi_{S}^{\alpha / 2}+\phi_{R}^{S, \alpha / 2}\right) \\
\exp i\left(\phi_{P}^{\alpha / 2-\pi}+D \phi_{S}^{\alpha / 2-\pi}+\phi_{R}^{S, \alpha / 2}\right) & 0
\end{array}\right) C(\kappa) \\
& M_{O}=C(\kappa)\left(\begin{array}{cc}
\exp i\left(\phi_{P}^{\alpha / 2}+D \phi_{S}^{\alpha / 2}-\phi_{R}^{S, \alpha / 2}\right) & 0 \\
0 & \exp i\left(\phi_{P}^{\alpha / 2-\pi}+D \phi_{S}^{\alpha / 2-\pi}+\phi_{R}^{S, \alpha / 2}\right)
\end{array}\right) \\
& M_{R}=-M_{C} C(\kappa)\left(\begin{array}{cc}
\operatorname{expi}\left(\phi_{P}^{-(\pi+\alpha)}+D \phi_{S}^{(\pi+\alpha)}+\phi_{R}^{C, \alpha}\right) & 0 \\
0 & \operatorname{expi}\left(\phi_{P}^{-(\alpha-\pi)}+D \phi_{S}^{(\alpha-\pi)}+\phi_{R}^{C, \alpha}\right)
\end{array}\right) C(\kappa) M_{C} \\
& M_{C}=\left(\begin{array}{cc}
\exp i\left(\phi_{P}^{(\pi+\alpha) / 2}+D \phi_{S}^{(\pi+\alpha) / 2}+\phi_{R}^{C, \alpha / 2}\right) & 0 \\
0 & \left.\operatorname{expi(} \phi_{P}^{(\alpha-\pi) / 2}+D \phi_{S}^{(\alpha-\pi) / 2}+\phi_{R}^{C, \alpha / 2}\right)
\end{array}\right) \\
& C(\kappa)=\frac{i}{\sqrt{\kappa}}\left(\begin{array}{cc}
\sqrt{1-\kappa} & -1 \\
1 & -\sqrt{1-\kappa}
\end{array}\right) \\
& \phi_{P}^{\theta}=\omega n R \theta / c ; \quad \phi_{S}^{\theta}=\omega \Omega R^{2} \theta / c^{2} ; \quad \phi_{R}^{S, \theta}=\omega \Omega R \tilde{R}_{0} \sin (\theta) / c^{2} ; \quad \phi_{R}^{C, \theta}=\omega \Omega R \tilde{R}_{0} \cos (\theta) / c^{2},
\end{aligned}
$$

where $M_{I}, M_{O}$, and $M_{R}$ are, respectively, the transfer matrices for the input section, the output section, and the recurring 




FIG. 2. Phase accumulation in a rotating microring resonator.

section, $D=1$ for the signal propagating with the device rotation and -1 for the signal counter propagating to the device rotation. $\alpha$ is the angle between adjacent microrings (see Fig. 1). The overall transfer matrix connecting the input and output of the CROW is, therefore, given by

$$
M_{\mathrm{CCW}}=M_{O}\left(M_{R}\right)^{(N-1) / 2} M_{I},
$$

where $N$ is the number of microrings comprising the CROW which must be odd for the configuration illustrated in Fig. 1.

Equations (5), (4), and (1) allow us to calculate the output signal $B_{r}$ for various parameters. A closer inspection of (4) allows us to eliminate some of the terms because we are not interested in the complete transmission function of the CROW but rather in the phase difference between the two paths. The phase terms proportional to $\tilde{R}_{0}$ can be rewritten as unit matrices multiplied by a common phase factor. Since this phase factor is identical for both paths, it has no effect on the outcome of (1), and therefore, the output signal $B_{r}$ is independent of $\tilde{R}_{0}$. This is an important conclusion because $\tilde{R}_{0}$ defines the area of the effective CROW ring. The Sagnac sensitivity in conventional waveguide loops is directly related to the area of the loop, thus we cannot analyze this effect in the CROW loop simply by assuming an effective ring waveguide with the dispersion relation of a CROW. It should be noted that the rotation induced phase due to the waveguide sections connecting the $3 d B$ coupler to the CROW is neglected because it can be made significantly smaller than that of the CROW.

Figure 3(a) depicts the output intensity $\left|B_{r}\right|^{2}$ as a function of $\Omega$ for CROWs with different number of resonators. The parameters of these CROWs are defined in the figure caption. As can be expected, the output intensity increases with $\Omega$ and the responsivity (slope) increases with the number of microrings. For the rotation-sensing application, a steeper slope is advantageous because it corresponds to higher sensitivity, i.e., the ability to detect slower rotation rates. Figs. 3(b)-3(d) show the relative responsivity of the CROW loop for a varying number of rings (3b), coupling coefficient (3c), and the microrings radius (3d). The responsivity increases with increasing number of microrings $N$, smaller coupling coefficient $k$, and larger microrings' radius $R$.

It is worthy to quantify some of these trends because they reveal the inherent differences between the Sagnac effect in CROWs and in conventional waveguides. Figure 3(b) shows a quadratic fit to the dependence of the responsivity on the number of rings comprising the CROW. The fit indicates that the responsivity of a closedloop CROW, $S_{N}$, consisting of $N$ microrings is related to that of a single ring according to $S_{N}=(N+1)^{2} S_{1} / 4$. It is well known that the slope of the output signal of a single- (a)

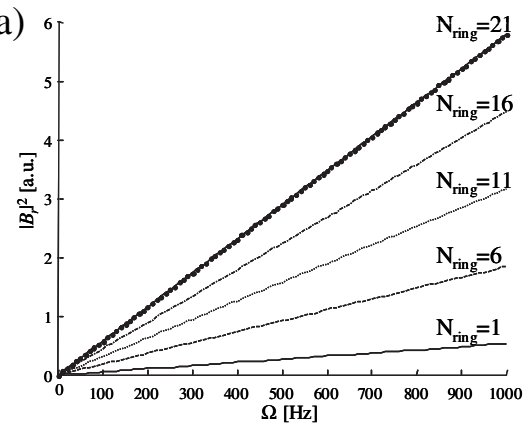

(c)



(b)

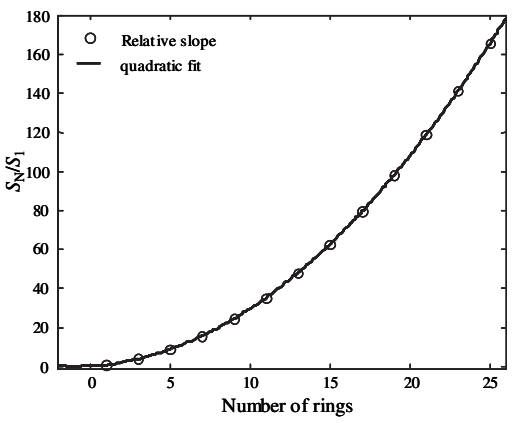

(d)

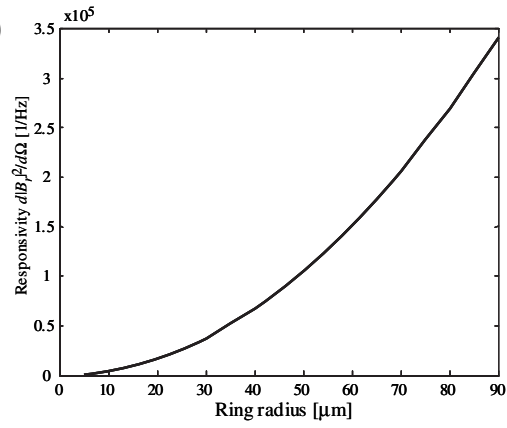

FIG. 3. (a) Output signal intensity as a function of the structure angular velocity for various numbers of rings, $R=$ $25 \mu \mathrm{m}, \kappa=0.01$. (b) Dependence of the relative sensitivity on the number of microrings, $\quad R=25 \mu \mathrm{m}, \quad \kappa=0.01$. Dependence of the sensitivity on: (c) the coupling coefficient ( $R=$ $25 \mu \mathrm{m}, N=9)$ and on (d) the microrings' radius $(N=9, \kappa=0.01)$. 




FIG. 4. The impact of the quality factor on the sensitivity. $R=$ $25 \mu \mathrm{m}, N=9, \kappa=0.03$.

ring device is proportional to the ring area [12], and therefore, the responsivity of the CROW-based device is proportional to the total area of the microrings composing it. Alternatively, it can be shown that the responsivity of the CROW gyro is proportional to the square effective length of the CROW, $L_{\text {eff }}=\pi R N /|\kappa|[7,8]$. In the interesting limit of $\kappa=1$ where the CROW loop is reduced to a "conventional" waveguide loop, this result coincides with the conventional expression for the responsivity because $L^{2}=4 \pi A_{\text {loop }}$. It should be emphasized that, unlike the conventional Sagnac effect, the overall area circumscribed by the CROW does not affect the output signal. This result, which clearly demonstrates the difference between the Sagnac effect in conventional and in CROWs, is interesting and, to some extent, counterintuitive because one might expect the Sagnac effect contributions from adjacent microrings to cancel each other. Figure 3(c) also compares the numerically calculated responsivity according to (5) and an analytic expression based on the derived responsivity of a single microring, and the quadratic dependence of the responsivity on the number of microrings in the CROW.

For practical applications, the CROW-based gyroscope exhibits several inherent advantages compared to conventional Sagnac loops: (1) The dependence of the gyro output signal on the interring coupling allows the enhancement of the device sensitivity without requiring larger area; (2) the independence of the responsivity of the CROW gyro on $\tilde{R}_{0}$ indicates that the arrangement of microrings comprising the CROW is insignificant, and thus, enables a more efficient utilization of the chip area.

In order to take advantage of the complete capacity of the CROW gyro it is desired that all cavities have the same resonance frequency. In practice, when such a structure is fabricated, there could be some deviations between the resonances of the cavities due to fabrication errors. However, this problem can be overcome by post-tuning the cavities after the fabrication using, e.g., the electro-optic or thermo-optic effects.

The ultimate limiting factor of the ability of a rotation sensor to detect slow angular velocity is the minimal detectable output power $\left|B_{r}\right|^{2}$ which is shot noise limited. While ideal microring resonators are lossless, when light propagates in real resonators it experiences propagation loss that can be included in our analysis by introducing an imaginary part to the index of refraction in (4). The propagation loss decreases the output signal and reduces the responsivity $d\left|B_{r}\right|^{2} / d \Omega$ of the rotation sensor. Figure 4 shows the responsivity of a CROW rotation sensor as a function of the resonators' quality factor $(Q)$. As shown in the figure, for resonators with $Q>10^{7}$, the influence of the propagation loss is negligible and has small effect on the device response. This transition occurs when the intrinsic losses of the cavities become smaller than the "coupling" losses (i.e., $\left.Q_{\text {int }} \gg Q_{\text {loaded }}\right)$. Since high- $Q\left(>10^{7}\right)$ singlemode, planar-technology-based microring resonators are being fabricated by many research groups [13], the propagation losses in the cavities do not limit significantly the sensitivity of the CROW rotation sensor.

J.S. is grateful to Beny Steinberg (Tel-Aviv University) for stimulating discussions and useful comments. The support of DARPA is gratefully acknowledged.

*Electronic address: koby@caltech.edu

[1] E. J. Post, Rev. Mod. Phys. 39, 475 (1967).

[2] H. J. Arditty and H. C. Lefèvre, Opt. Lett. 6, 401 (1981).

[3] C. Liu et al., Nature (London) 409, 490 (2001).

[4] D. F. Phillips et al., Phys. Rev. Lett. 86, 783 (2001).

[5] M. S. Bigelow et al., Phys. Rev. Lett. 90, 113903 (2003).

[6] A. V. Turukhin et al., Phys. Rev. Lett. 88, 023602 (2002).

[7] A. Yariv et al., Opt. Lett. 24, 711 (1999); A. Melloni et al., Opt. Quantum Electron. 35, 365 (2003); M. F. Yanik and S. Fan, Phys. Rev. Lett. 92, 083901 (2004); M. F. Yanik et al., Phys. Rev. Lett. 93, 233903 (2004); J. Scheuer et al., Opt. Photonics News 16, 36 (2005).

[8] J.E. Heebner et al., J. Opt. Soc. Am. B 21, 1818 (2004); J. E. Heebner et al., Phys. Rev. E 65, 036619 (2002).

[9] U. Leonhardt and P. Piwnitski, Phys. Rev. A 62, 055801 (2000).

[10] B. Z. Steinberg, Phys. Rev. E 71, 056621 (2005).

[11] A. B. Matsko et al., Opt. Commun. 233, 107 (2004).

[12] S. Ezekiel et al., in Optical Fiber Rotation Sensing, edited by W. K. Burns (Academic Press Inc., Boston, 1994).

[13] M.L. Gorodetsky et al., Opt. Lett. 21, 453 (1996); T. J. Kippenberg et al., Appl. Phys. Lett. 85, 6113 (2004); K. J. Vahala, Nature (London) 424, 839 (2003). 